\title{
Return to intended oncologic treatment after surgery for malignant pleural mesothelioma
}

\author{
David B. Nelson, MD, MSc, ${ }^{\mathrm{a}}$ David C. Rice, MB, BCh, ${ }^{\mathrm{a}}$ Kyle G. Mitchell, MD, ${ }^{\mathrm{a}}$ Anne S. Tsao, MD, ${ }^{\mathrm{b}}$ \\ Daniel R. Gomez, MD, ${ }^{\mathrm{c}}$ Boris Sepesi, MD, ${ }^{\mathrm{a}}$ and Reza J. Mehran, MD, MSc ${ }^{\mathrm{a}}$
}

\section{ABSTRACT}

Objective: Trimodality therapy may prolong survival for patients with resectable malignant pleural mesothelioma. However, many patients are unable to complete therapy. We sought to identify risk factors for failing to complete adjuvant intensity-modulated radiation therapy after cytoreduction for malignant pleural mesothelioma.

Methods: We performed a single-institution review of those who received an extrapleural pneumonectomy or pleurectomy/decortication for malignant pleural mesothelioma from 2004 to 2017. Multivariable logistic regression was used to assess preoperative or intraoperative risk factors associated with failing to complete adjuvant intensity-modulated radiation therapy.

Results: A total of 160 patients were identified, among whom 94 (59\%) received an extrapleural pneumonectomy and 66 (41\%) received a pleurectomy/ decortication. Adjuvant intensity-modulated radiation therapy was completed among 105 patients $(66 \%)$. Reasons for failing to complete adjuvant intensitymodulated radiation therapy included mortality (19), dose constraints (21), postoperative morbidity or delayed recovery (11), and refused or unknown status (4). On multivariable analysis, American Society of Anesthesiologists $3+$ classification $(P=.002)$ and smoking history $(P=.022)$ were associated with failure to complete adjuvant intensity-modulated radiation therapy, whereas forced expiratory volume in 1 second $70 \%$ or less of predicted and pStage 4 (T4) were significant on univariable analysis only. Other factors, including extrapleural pneumonectomy or pleurectomy/decortication, margin status, age, and histology,

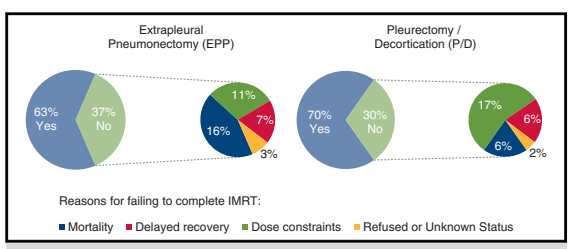

Rate of completing adjuvant IMRT.

\section{Central Message}

After cytoreductive surgery for MPM, only $66 \%$ of patients were able to complete adjuvant IMRT as intended.

\section{Perspective}

We identified that worse baseline comorbidity status, as manifested by American Society of Anesthesiologists classification and smoking history, was associated with decreased odds of completing adjuvant IMRT. However, other factors, including receiving an EPP or margin status, were not associated with decreased use of adjuvant IMRT.

See Commentaries on pages 930 and 932 . were not associated with receiving adjuvant intensity-modulated radiation therapy.

Conclusions: Many patients are unable to complete adjuvant intensity-modulated radiation therapy after cytoreduction. Failure to complete adjuvant intensity-modulated radiation therapy was associated with worse preoperative comorbidity, but not the type of surgery or margin status. (J Thorac Cardiovasc Surg 2019;158:924-9)

Malignant pleural mesothelioma (MPM) is an aggressive and rapidly fatal malignancy. Median survival after diagnosis remains 10 months, despite therapeutic advancements. ${ }^{1}$ Several phase II observational studies have shown prolonged survival with trimodality therapy, which consists of neoadjuvant chemotherapy, cytoreductive surgery, and

From the Departments of ${ }^{\mathrm{a}}$ Thoracic and Cardiovascular Surgery, ${ }^{\mathrm{b}}$ Medical Oncology, and ${ }^{c}$ Radiation Oncology, The University of Texas MD Anderson Cancer Center, Houston, Tex.

This study was funded by generous philanthropic donations from the Mason Family, anonymous donors, and departmental support.

Institutional Review Board Protocol PA17-1010, Approval Date January 18, 2018.

Read at The American Association for Thoracic Surgery Thoracic Summit, New York, New York, October 12-13, 2018.

Received for publication Sept 26, 2018; revisions received Feb 12, 2019; accepted for publication Feb 22, 2019. adjuvant radiation..$^{2-6}$ Excellent rates of local control can be achieved with extrapleural pneumonectomy (EPP) and adjuvant intensity-modulated radiation therapy (IMRT) with minimal toxicity to the remaining lung, ${ }^{7-12}$ and adjuvant IMRT can also be delivered with the lung in situ. $^{13-16}$

\footnotetext{
Address for reprints: Reza J. Mehran, MD, MSc, Department of Thoracic and Cardiovascular Surgery, The University of Texas MD Anderson Cancer Center, 1515 Holcombe Blvd, Unit 1489, Houston, TX 77030 (E-mail: rmehran@ mdanderson.org). 0022-5223/\$36.00

Copyright (C) 2019 Published by Elsevier Inc. on behalf of The American Association for Thoracic Surgery

https://doi.org/10.1016/j.jtcvs.2019.02.129
} 

Abbreviations and Acronyms
$\mathrm{EPP}=$ extrapleural pneumonectomy
MPM = malignant pleural mesothelioma
$\mathrm{P} / \mathrm{D}=$ pleurectomy/decortication

To view the AATS Meeting Webcast, see the URL next to the webcast thumbnail.

However, many patients are unable to complete intended therapy. Risks of postoperative morbidity or mortality after cytoreductive surgery remain high and present as a major burden. In a large nationwide study among 3058 surgical patients, 90-day mortality after cytoreductive surgery for MPM was found to be $15.5 \% .{ }^{17}$ Results from a phase II trial showed that of 77 patients given induction chemotherapy, only $40(52 \%)$ were able to complete cytoreductive surgery and adjuvant radiotherapy. ${ }^{2}$

At our institution, our standard treatment practice is to administer adjuvant IMRT after cytoreduction (Figure 1). The objective of this study was to determine factors that were associated with failing to complete adjuvant IMRT. We hypothesized that failing to complete adjuvant IMRT could be predicted using preoperative or intraoperative information. Our purpose with this retrospective review was to identify patients who would be unable to complete adjuvant IMRT as intended, and therefore be unable to achieve optimal locoregional control.

\section{MATERIAL AND METHODS \\ Patient Selection}

This study was approved by the Institutional Review Board at The University of Texas MD Anderson Cancer Center, which waived the requirement for informed consent. A prospectively maintained departmental database was queried to identify patients who underwent a pleurectomy/decortication (P/D) or EPP for MPM from January 1, 2004, to September 31, 2017. Patients who received an extended P/D were included as a P/D for the purposes of this analysis. Patients who received an exploratory thoracotomy with a partial P/D without an attempt at maximal cytoreduction were excluded from analysis. We also excluded patients for whom there was no intent to provide adjuvant IMRT, which occurred most often after pleurectomy/decortication earlier in the study period $(\mathrm{n}=5)$.

\section{Preoperative Evaluation and Surgical Details}

All patients were staged with a positron emission tomography/ computed tomography, bronchoscopy, laparoscopy, and mediastinoscopy. Patients with N2 disease or those with evidence of metastases are excluded from consideration for cytoreductive surgery. Pulmonary function was evaluated with spirometry and quantitative ventilation/perfusion scanning. Patients who received a P/D were required to be physiologically capable of also receiving EPP. The operative choice of performing an EPP or P/D was according to the discretion of the operating surgeon. Carefully selected patients with biphasic histology were considered for cytoreduction. Neoadjuvant chemotherapy regimen consisted of a platinum-based chemotherapy doublet with pemetrexed.

\section{Radiation Therapy}

The IMRT protocol was delivered as previously described. ${ }^{7}$ The dose prescribed to the planned target volume was 45 to $50 \mathrm{~Gy}$ in 25 daily fractions, with a boost to 55 to $60 \mathrm{~Gy}$ provided for positive margins or areas at high risk for residual tumor.

\section{Variable Definitions}

All covariates were collected and maintained in a prospectively maintained departmental database with additional chart review performed. The American Joint Committee on Cancer Seventh Edition was used to define pathologic stage. Epithelioid, biphasic, or sarcomatoid histologic subtype reflected findings among the final pathologic specimen after resection. Weight loss and smoking history were abstracted from preoperative history and physical intake forms. Weight loss represented any self-reported weight loss by the patient. Postoperative complications were defined according to previously published criteria. ${ }^{18}$ The reason for not receiving adjuvant IMRT was determined by the treating radiation oncologist and abstracted from statements within clinic progress notes. Reasons included delayed recovery or mobidity, patient refusal or undocumented reason, and dose constraints. Large areas of remaining or recurrent bulky tumor observed on imaging were often unable to be treated to limit toxicity to nearby organs. In the case of patient death before the outpatient radiation oncology follow-up, the patient was deemed to have not completed adjuvant IMRT because of mortality. Patients who started adjuvant IMRT and failed to complete the treatment were considered to have not received adjuvant IMRT for the purposes of this study.

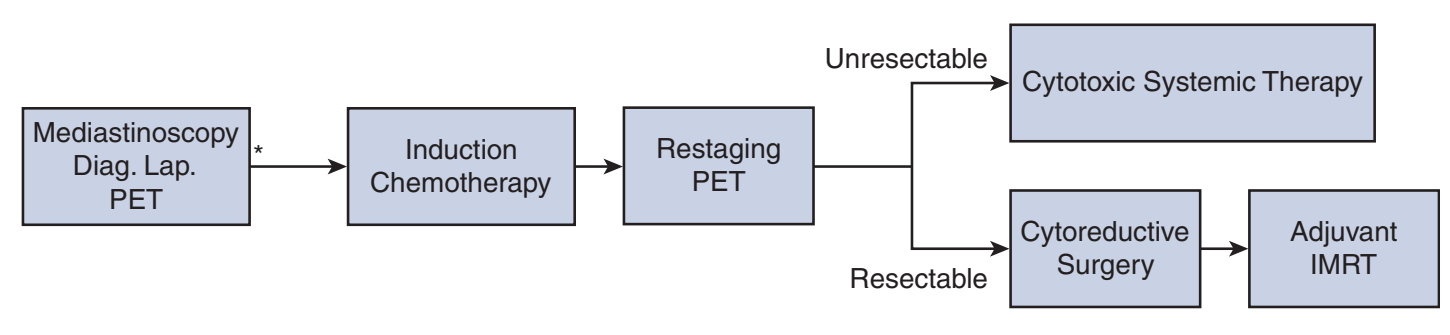

* No evidence of N2, N3, or T4 disease

FIGURE 1. Treatment strategy framework at MD Anderson Cancer Center (2018). Patients typically receive staging with mediastinoscopy, diagnostic laparoscopy, and positron emission tomography followed by induction chemotherapy. Diag. Lap, Diagnostic laparoscopy; IMRT, intensity-modulated radiation therapy; PET, positron emission tomography. 

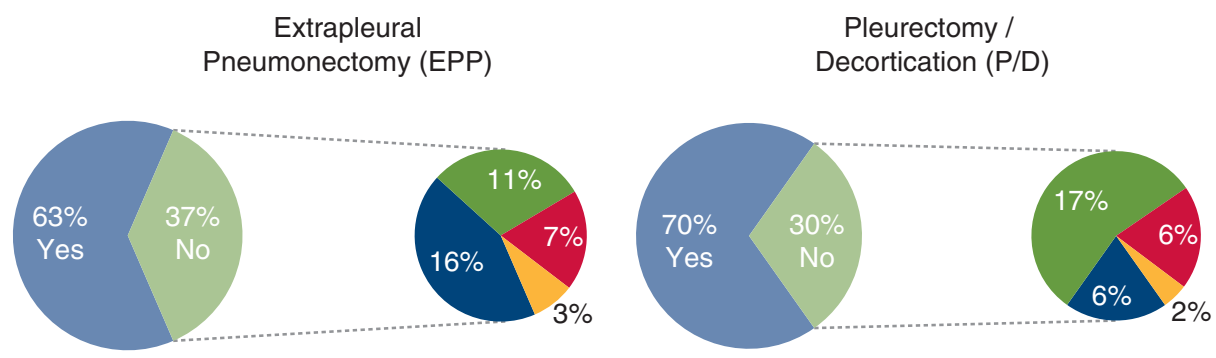

Reasons for failing to complete IMRT:

- Mortality - Delayed recovery $\square$ Dose constraints $\square$ Refused or Unknown Status

FIGURE 2. Rate of completing adjuvant IMRT according to type of surgery. IMRT, Intensity-modulated radiation therapy.

\begin{abstract}
Statistical Methods
Chi-square or Fisher exact test was used to compare frequency of categoric variables. Student $t$ test or Mann-Whitney test was used to evaluate for differences in continuous variables, where appropriate. Cochrane-Armitage test was used to evaluate for trends in adjuvant IMRT use during the study duration. A univariable logistic regression model was used to evaluate for factors associated with completion of adjuvant IMRT. Covariates with $P$ less than .25 or those of clinical interest were evaluated for multivariable analysis. Robust standard errors were used for regression analysis. The final model was selected using backward selection criteria. All tests were 2 sided. All statistics were performed using STATA version 14.2 (StataCorp LP, College Station, Tex).
\end{abstract}

\section{RESULTS}

\section{Patient Characteristics}

A total of 160 patients were identified, among whom $94(59 \%)$ received an EPP and $66(41 \%)$ received a P/D. After resection, only 15 patients $(9 \%)$ had grossly positive margins. Most patients had pathologically stage III or IV stage, despite being aggressively staged with mediastinoscopy. The 30-day mortality was higher after EPP $(6 \%, 6 / 94)$ versus P/D $(0 \%, 0 / 66)$ $(P=.038)$. However, the 90 -day mortality was not higher after EPP $(13 \%, 12 / 94)$ versus P/D (4/66, 6\%) $(P=.130)$.

Adjuvant IMRT was completed among 105 patients (66\%) (Figure 2). Characteristics of those able to complete adjuvant IMRT are shown in Table 1 . The median number of days to complete all intended adjuvant IMRT was 102 days postoperatively (interquartile range, 90-116 days). Reasons for not being able to complete adjuvant IMRT were as follows: $19(12 \%)$ mortality, $21(13 \%)$ dose constraints, $11(7 \%)$ postoperative morbidity or delayed recovery, and $4(3 \%)$ refused or unknown status (Table 2$)$. There were no trends identified in the rate of receiving adjuvant IMRT over the study duration period $(P=.927)$. The rate of receiving adjuvant IMRT among those who experienced cardiovascular or pulmonary complications was approximately half of those who did not $(P<.01$ for all, Table 1).

\section{Prognostic Factors Toward Completing Adjuvant Intensity-Modulated Radiation Therapy}

A prognostic model to identify preoperative or intraoperative factors that were associated with failure to receive adjuvant IMRT is shown in Table 3. Although suffering from complications was strongly associated with failing to receive adjuvant IMRT, we restricted this analysis to information that would be available preoperatively or intraoperatively. Pathologic stage and histologic subtype were included because of inaccuracy with clinical staging of mesothelioma. ${ }^{19-21}$

Preoperative American Society of Anesthesiologists $3+$ classification was strongly associated with failure to receive adjuvant IMRT (hazard ratio, $0.27 ; 95 \%$ confidence interval, $0.12-0.63 ; P=.002$ ), as was smoking history (hazard ratio, $0.42 ; 95 \%$ confidence interval, 0.20-0.88; $P=.022)$. Patients with forced expiratory volume in 1 second $70 \%$ or less than predicted and pathologic stage IV (T4) showed a decreased likelihood of completing adjuvant IMRT on univariable analysis; however, these differences disappeared after multivariable adjustment. Nonepithelioid subtype, older age, and preoperative weight loss were not associated with failure to complete adjuvant IMRT. In addition, the rate of completing adjuvant radiation was not different among those who received EPP or P/D, nor was it different among patients who received an $\mathrm{R} 0 / \mathrm{R} 1$ resection versus those who received an $\mathrm{R} 2$ resection.

\section{DISCUSSION}

Many patients are unable to complete adjuvant IMRT after cytoreductive surgery for MPM, with one-third of patients discontinuing treatment. Our results parallel those of several phase II trials for trimodality therapy. We identified that worse preoperative comorbidity status, as identified by American Society of Anesthesiologists status and smoking history, was associated with decreased likelihood of receiving adjuvant IMRT. The importance of perioperative complications toward receiving adjuvant treatment is underscored by the observation that the rate 
TABLE 1. Characteristics of patients able to complete adjuvant intensity-modulated radiation therapy

\begin{tabular}{|c|c|c|c|}
\hline & $\begin{array}{c}\text { Adjuvant } \\
\text { IMRT } \\
(\mathbf{n}=\mathbf{1 0 5})^{*}\end{array}$ & $\begin{array}{c}\text { No } \\
\text { adjuvant } \\
\text { IMRT } \\
(\mathbf{n}=\mathbf{5 5})^{*} \\
\end{array}$ & $\begin{array}{c}P \\
\text { value }\end{array}$ \\
\hline Age, mean (SD), y & $61.5 \pm 8.25$ & $63.0 \pm 7.80$ & .255 \\
\hline Male sex & $80(76 \%)$ & $43(78 \%)$ & .777 \\
\hline Weight loss & $22(21 \%)$ & $8(15 \%)$ & .324 \\
\hline Smoking history & $52(50 \%)$ & $39(71 \%)$ & .013 \\
\hline $\begin{array}{l}\text { ASA classification } \\
1 \\
2 \\
3 \\
4\end{array}$ & $\begin{array}{c}8(8 \%) \\
36(34 \%) \\
60(57 \%) \\
1(1.0 \%)\end{array}$ & $\begin{array}{c}0 \\
8(15 \%) \\
44(80 \%) \\
3(5 \%)\end{array}$ & .001 \\
\hline$\%$ predicted FEV1, mean $(\mathrm{SD})$ & $78.1 \pm 16.8$ & $73.3 \pm 14.6$ & .075 \\
\hline Neoadjuvant chemotherapy & $72(69 \%)$ & $36(65 \%)$ & .689 \\
\hline $\begin{array}{l}\text { Resection type } \\
\text { P/D } \\
\text { EPP }\end{array}$ & $\begin{array}{l}46(44 \%) \\
59(56 \%)\end{array}$ & $\begin{array}{l}20(36 \%) \\
35(63 \%)\end{array}$ & .364 \\
\hline $\begin{array}{l}\text { Histologic subtype } \\
\text { Epithelioid } \\
\text { Biphasic } \\
\text { Sarcomatoid }\end{array}$ & $\begin{array}{c}83(79 \%) \\
18(17 \%) \\
4(4 \%)\end{array}$ & $\begin{array}{c}39(71 \%) \\
15(27 \%) \\
1(2 \%)\end{array}$ & .277 \\
\hline $\begin{array}{l}\text { Margin status } \\
\text { R0/R1 } \\
\text { R2 }\end{array}$ & $\begin{array}{c}97(92 \%) \\
8(8 \%)\end{array}$ & $\begin{array}{r}48(87 \%) \\
7(13 \%)\end{array}$ & .292 \\
\hline $\begin{array}{l}\text { Pathologic stage } \\
\quad \text { Stage I } \\
\text { Stage II } \\
\text { Stage III } \\
\text { Stage IV }\end{array}$ & $\begin{array}{c}6(6 \%) \\
16(15 \%) \\
56(53 \%) \\
27(26 \%)\end{array}$ & $\begin{array}{c}2(4 \%) \\
4(7 \%) \\
26(47 \%) \\
23(42 \%)\end{array}$ & .145 \\
\hline Length of stay, median (IQR) & $8(6-11)$ & $10(7-21)$ & .001 \\
\hline Pulmonary complications & $39(37 \%)$ & $34(62 \%)$ & .003 \\
\hline Cardiovascular complications & $38(36 \%)$ & $34(62 \%)$ & .002 \\
\hline Readmission to ICU postoperatively & $5(5 \%)$ & $8(15 \%)$ & .063 \\
\hline
\end{tabular}

IMRT, Intensity-modulated radiation therapy; $S D$, standard deviation; $A S A$, American Society of Anesthesiologists; $F E V I$, forced expiratory volume in 1 second; $P / D$, pleurectomy/decortication; $E P P$, extrapleural pneumonectomy; $I Q R$, interquartile range; $I C U$, intensive care unit. *Indicates $\mathrm{N}(\%)$ unless otherwise stated.

of completing adjuvant IMRT was approximately half among those who experienced cardiovascular or pulmonary complications. However, in our population, we did not observe that receiving an EPP or P/D influenced the rate of receiving adjuvant IMRT. In addition, the extent of resection, whether an R0/R1 or R2, was also not associated with the likelihood of receiving adjuvant IMRT.

Median survival has been shown to be significantly higher among those who are able to complete trimodality therapy. In a prospective phase II trial, 16.8-month median survival was observed among the entire intent-to-treat study
TABLE 2. Reasons for failing to complete adjuvant intensitymodulated radiation therapy

\begin{tabular}{lccc}
\hline & $\begin{array}{c}\text { EPP } \\
(\mathbf{N}=\mathbf{9 4})\end{array}$ & $\begin{array}{c}\text { P/D } \\
(\mathbf{N}=\mathbf{6 6})\end{array}$ & $\begin{array}{c}\boldsymbol{P} \\
\text { value }\end{array}$ \\
\hline Mortality & $15(16 \%)$ & $4(6 \%)$ & .277 \\
$\begin{array}{l}\text { Dose constraints } \\
\begin{array}{l}\text { Postoperative morbidity } \\
\text { or delayed recovery }\end{array}\end{array}$ & $10(11 \%)$ & $11(17 \%)$ & \\
$\begin{array}{l}\text { Refused or unknown status } \\
\text { Refo) }\end{array}$ & $4(6 \%)$ & \\
\hline
\end{tabular}

$E P P$, Extrapleural pneumonectomy; $P / D$, pleurectomy/decortication.

cohort that received induction chemotherapy, and was extended to 29.1 months among those who were able to complete EPP and adjuvant radiation per protocol. ${ }^{2}$ It is unclear whether survivorship bias, which results from the "survivors" and those with more favorable disease biology preferentially finishing therapy, explains these results. In addition to concerns regarding survivorship bias, the patients who are unable to complete trimodality therapy represent whose who had an adverse event that was significant enough to result in discontinued treatment. Determining the rate of returning to intended oncologic treatment and factors that may predict completing intended oncologic treatment may lead to important advancements in patient selection for cytoreductive surgery.

\section{Study Limitations}

Limitations of this study include the risk of residual confounding in this retrospective study. There may also be important unmeasured confounding relationships that were not accounted for. In addition, our outcomes represent those of an experienced and high-volume academic center and may not be generalizable to other institutions. Patients who receive cytoreductive surgery at our institution represent carefully selected surgical candidates. Last, although this study represents a relatively large series for mesothelioma, the power of this study is an additional limitation. Although there were no statistically significant differences between EPP or P/D in the reason for failing to complete adjuvant IMRT, this may represent a type II error.

\section{CONCLUSIONS}

Because of the high rate of failing to complete subsequent adjuvant therapy after cytoreduction for MPM, our findings support highly selective patient selection. Patients with higher baseline comorbidity should not be considered for cytoreduction. We were unable to show that the margin status and receiving an EPP or P/D had any relationship with receiving adjuvant IMRT. 
TABLE 3. Univariable and multivariable preoperative or intraoperative factors associated with completing adjuvant intensitymodulated radiation therapy

\begin{tabular}{|c|c|c|c|c|}
\hline & \multicolumn{2}{|c|}{ Univariable } & \multicolumn{2}{|c|}{ Multivariable } \\
\hline & $\begin{array}{c}\text { OR } \\
(95 \% \mathbf{C I})\end{array}$ & $\begin{array}{c}P \\
\text { value }\end{array}$ & $\begin{array}{c}\text { OR } \\
(95 \% \mathrm{CI})\end{array}$ & $\begin{array}{c}P \\
\text { value }\end{array}$ \\
\hline Age (per y) & $0.98(0.94-1.02)$ & .262 & & \\
\hline Sex, female & $1.12(0.51-2.45)$ & .777 & & \\
\hline Weight loss & $1.56(0.64-3.78)$ & .328 & & \\
\hline Smoking history & $0.40(0.20-0.81)$ & .011 & $0.42(0.20-0.88)$ & .022 \\
\hline $\begin{array}{l}\text { ASA classification } \\
1-2 \text { (reference) } \\
3+\end{array}$ & $0.24(0.10-0.55)$ & .001 & $0.27(0.12-0.63)$ & .002 \\
\hline $\begin{array}{l}\text { FEV1 } \\
\quad>70 \% \\
\quad(\text { reference }) \\
\leq 70 \%\end{array}$ & $0.50(0.26-0.98)$ & .043 & $0.55(0.27-1.12)$ & .103 \\
\hline $\begin{array}{l}\text { Histologic subtype } \\
\text { Epithelioid } \\
\text { (reference) }\end{array}$ & - & & & \\
\hline Nonepithelioid & $0.65(0.31-1.37)$ & .254 & & \\
\hline $\begin{array}{l}\text { Induction } \\
\text { chemotherapy }\end{array}$ & $1.15(0.58-2.31)$ & .690 & & \\
\hline $\begin{array}{l}\text { Resection type } \\
\text { P/D (reference) } \\
\text { EPP }\end{array}$ & $0.73(0.37-1.44)$ & .366 & & \\
\hline $\begin{array}{l}\text { Margin status } \\
\text { R0-R1 } \\
\text { (reference) } \\
\text { R2 }\end{array}$ & - & & & \\
\hline R2 & 6) & .299 & & \\
\hline $\begin{array}{l}\text { Pathologic stage } \\
\text { Stage I-II } \\
\text { (reference) }\end{array}$ & - & & - & \\
\hline $\begin{array}{l}\text { Stage III } \\
\text { Stage IV (T4) }\end{array}$ & 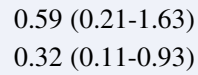 & $\begin{array}{l}.306 \\
.036\end{array}$ & $\begin{array}{l}0.79(0.25-2.52) \\
0.43(0.13-1.42)\end{array}$ & $\begin{array}{l}.697 \\
.165\end{array}$ \\
\hline
\end{tabular}

Postoperative complications, ICU readmission, and prolonged length of stay were statistically significant on univariable analysis but were not included because of not being known to the treating provider preoperatively or intraoperatively. $O R$, Odds ratio; $C I$, confidence interval; $A S A$, American Society of Anesthesiologists; FEVI, forced expiratory volume in 1 second; $P / D$, pleurectomy/decortication; $E P P$, extrapleural pneumonectomy.

\section{Webcast}

You can watch a Webcast of this AATS meeting presentation by going to: https://aats.blob.core.windows. net/media/ITSOS18/GS-12-1415-1430-Nelson-720p.mp4.

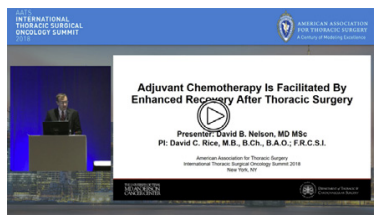

\section{Conflict of Interest Statement}

Authors have nothing to disclose with regard to commercial support.

\section{References}

1. Nelson DB, Rice DC, Niu J, Atay SM, Vaporciyan AA, Antonoff MB, et al. Predictors of trimodality therapy and trends in therapy for malignant pleural mesothelioma. Eur J Cardiothorac Surg. 2018;53:960-6.

2. Krug LM, Pass HI, Rusch VW, Kindler HL, Sugarbaker DJ, Rosenzweig KE, et al. Multicenter phase II trial of neoadjuvant pemetrexed plus cisplatin followed by extrapleural pneumonectomy and radiation for malignant pleural mesothelioma. J Clin Oncol. 2009;27:3007-13.

3. Van Schil PE, Baas P, Gaafar R, Maat AP, Van de Pol M, Hasan B, et al. Trimodality therapy for malignant pleural mesothelioma: results from an EORTC phase II multicentre trial. Eur Respir J. 2010;36:1362-9.

4. Rea F, Adolfo F, Giuseppe M, Lorenzo S, Martino DT, Anna C, et al. Phase II trial of neoadjuvant pemetrexed plus cisplatin followed by surgery and radiation in the treatment of pleural mesothelioma. BMC Cancer. 2013;13:22.

5. de Perrot M, Feld R, Cho BC, Bezjak A, Anraku M, Burkes R, et al. Trimodality therapy with induction chemotherapy followed by extrapleural pneumonectomy and adjuvant high-dose hemithoracic radiation for malignant pleural mesothelioma. J Clin Oncol. 2009;27:1413-8.

6. Rea F, Marulli G, Bortolotti L, Breda C, Favaretto AG, Loreggian L, et al. Induction chemotherapy, extrapleural pneumonectomy (EPP) and adjuvant hemi-thoracic radiation in malignant pleural mesothelioma (MPM): feasibility and results. Lung Cancer. 2007;57:89-95.

7. Gomez DR, Hong DS, Allen PK, Welsh JS, Mehran RJ, Tsao AS, et al Patterns of failure, toxicity, and survival after extrapleural pneumonectomy and hemithoracic intensity-modulated radiation therapy for malignant pleural mesothelioma. J Thorac Oncol. 2013;8:238-45.

8. Rice DC, Stevens CW, Correa AM, Vaporciyan AA, Tsao A, Forster KM, et al. Outcomes after extrapleural pneumonectomy and intensity-modulated radiation therapy for malignant pleural mesothelioma. Ann Thorac Surg. 2007; 84:1685-92.

9. Ahamad A, Stevens CW, Smythe WR, Vaporciyan AA, Komaki R, Kelly JF, et al. Intensity-modulated radiation therapy: a novel approach to the management of malignant pleural mesothelioma. Int J Radiat Oncol Biol Phys. 2003;55: 768-75.

10. Miles EF, Larrier NA, Kelsey CR, Hubbs JL, Ma J, Yoo S, et al. Intensity-modulated radiotherapy for resected mesothelioma: the Duke experience. Int J Radiat Oncol Biol Phys. 2008;71:1143-50.

11. Krayenbuehl J, Oertel S, Davis JB, Ciernik IF. Combined photon and electron three-dimensional conformal versus intensity-modulated radiotherapy with integrated boost for adjuvant treatment of malignant pleural mesothelioma after pleuropneumonectomy. Int J Radiat Oncol Biol Phys. 2007;69:1593-9.

12. Chi A, Liao Z, Nguyen NP, Howe C, Gomez D, Jang SY, et al. Intensity-modulated radiotherapy after extrapleural pneumonectomy in the combined-modality treatment of malignant pleural mesothelioma. J Thorac Oncol. 2011;6:1132-41.

13. Pan HY, Jiang S, Sutton J, Liao Z, Chance WW, Frank SJ, et al. Early experience with intensity modulated proton therapy for lung-intact mesothelioma: a case series. Pract Radiat Oncol. 2015;5:e345-53.

14. Chance WW, Rice DC, Allen PK, Tsao AS, Fontanilla HP, Liao Z, et al. Hemithoracic intensity modulated radiation therapy after pleurectomy/ decortication for malignant pleural mesothelioma: toxicity, patterns of failure, and a matched survival analysis. Int J Radiat Oncol Biol Phys. 2015; 91:149-56.

15. Rosenzweig KE, Zauderer MG, Laser B, Krug LM, Yorke E, Sima CS, et al. Pleural intensity-modulated radiotherapy for malignant pleural mesothelioma. Int J Radiat Oncol Biol Phys. 2012;83:1278-83.

16. Minatel E, Trovo M, Polesel J, Baresic T, Bearz A, Franchin G, et al. Radical pleurectomy/decortication followed by high dose of radiation therapy for malignant pleural mesothelioma. Final results with long-term follow-up. Lung Cancer. 2014;83:78-82

17. Nelson DB, Rice DC, Niu J, Atay S, Vaporciyan AA, Antonoff M, et al. Long-term survival outcomes of cancer-directed surgery for malignant pleural mesothelioma: propensity score matching analysis. J Clin Oncol. 2017;35:3354-62.

18. Van Haren RM, Mehran RJ, Correa AM, Antonoff MB, Baker CM, Woodard TC, et al. Enhanced recovery decreases pulmonary and cardiac 
complications following thoracotomy for lung cancer. Ann Thorac Surg. 2018; 106:272-9.

19. Greillier L, Cavailles A, Fraticelli A, Scherpereel A, Barlesi F, Tassi G, et al. Accuracy of pleural biopsy using thoracoscopy for the diagnosis of histologic subtype in patients with malignant pleural mesothelioma. Cancer. 2007;110:2248-52.

20. Heelan RT, Rusch VW, Begg CB, Panicek DM, Caravelli JF, Eisen C. Staging of malignant pleural mesothelioma: comparison of CT and MR imaging. AJR Am J Roentgenol. 1999;172:1039-47.
21. Flores RM. The role of PET in the surgical management of malignant pleural mesothelioma. Lung Cancer. 2005;49(Suppl 1):S27-32.

Key Words: mesothelioma, IMRT, intensity modulated radiation therapy, extrapleural pneumonectomy, pleurectomy decortication 